\title{
Optical Conductivity of Mayenite: From Insulator to Metal
}

\author{
R. P. S. M. Lobo, ${ }^{* \dagger}$ N. Bontemps, ${ }^{\dagger}$ M. I. Bertoni, ${ }^{\ddagger}$ T. O. Mason, ${ }^{\ddagger}$ K. R. Poeppelmeier ${ }^{\dagger}$ A. J. Freeman, ${ }^{\ddagger}$ \\ M. S. Park, ${ }^{\S}$ and J. E. Medvedeva ${ }^{\S}$ \\ ${ }^{\dagger}$ PSL Research University; CNRS, UMR 8213; Sorbonne Universités, UPMC Univ. Paris 6, ESPCI-ParisTech, LPEM, 10 rue \\ Vauquelin, F-75231 Paris, Cedex 5, France \\ ${ }^{\ddagger}$ Northwestern University, Evanston, Illinois 60208, United States \\ $\S_{\text {Missouri University of Science and Technology, Rolla, Missouri 65409, United States }}$
}

ABSTRACT: Mayenite-based oxides $\left[12 \mathrm{CaO} \cdot 7 \mathrm{Al}_{2} \mathrm{O}_{3}\right]$ starting from $\left[\mathrm{Ca}_{24} \mathrm{Al}_{28} \mathrm{O}_{64}\right]^{4+}+2 \mathrm{O}^{2-}$ (insulator) and subsequently annealed so as to obtain $\left[\mathrm{Ca}_{24} \mathrm{Al}_{28} \mathrm{O}_{64}\right]^{4+}+4 \mathrm{e}^{-}$(metal) were studied by reflectance and transmission on seven samples with $\mathrm{dc}$ conductivities spanning the range $10^{-10}$ to $1500 \Omega^{-1} \mathrm{~cm}^{-1}$. Three of them are essentially insulating. The remaining display an increasing $\mathrm{dc}$ conductivity as the electron concentration increases. A set of phonons in the infrared below $0.15 \mathrm{eV}$ and an intense line in the ultraviolet at $6.5 \mathrm{eV}$ account for the optical conductivity of the insulating samples. As the samples become increasingly metallic, we observe an enhancement of spectral weight in the visible range. Simultaneously, the reflectance and the resulting optical conductivity develop a stronger component in the infrared, characteristic of mobile electrons. This electronic response appears to be strongly coupled to the phonons, as shown by their Fano profiles present in the metallic samples. Our results indicate that free carriers promote the

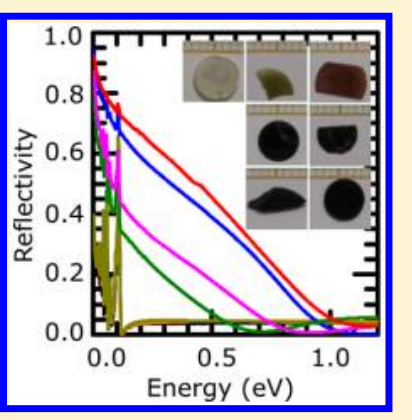
formation of polarons and that these two excitations contribute to the dc conducting properties of mayenites. This overall electronic picture is consistent with first principles calculations of the density of states in mayenites. The observed strong electron-phonon coupling may be of interest in view of the superconductivity properties found in the metallic samples.

\section{INTRODUCTION}

Mayenite, $\mathrm{Ca}_{12} \mathrm{Al}_{14} \mathrm{O}_{33}$, a well-known constituent of high alumina cements, was introduced in 1988 by Lacerda et al. as a high oxide conductor. ${ }^{1,2}$ Fourteen years later it was presented as a novel UV-activated electronic conductor. ${ }^{3}$ Since then, its applications as transparent conductor, catalyst, ionic conductor, radiation resistant material, electron emitter, and superconductor have been investigated. ${ }^{4-12} \mathrm{Ca}_{12} \mathrm{Al}_{14} \mathrm{O}_{33}$, a wideband-gap oxide, is a body-centered cubic crystal that belongs to the space group $I \overline{4} 3 d$ with a lattice parameter of $11.989 \AA^{13} \mathrm{It}$ is a cage-like structure with two formula units and 12 cages per unit cell. The diameter of each cage is approximately $5 \AA$. Openings of $3.5 \AA$ on the wall of the cages are believed to control the mass transport between the inside and outside of the cages. The chemical formula may be rewritten as $\left[\mathrm{Ca}_{24} \mathrm{Al}_{28} \mathrm{O}_{64}\right]^{4+}+2 \mathrm{O}^{2-}$, where the first term denotes the lattice framework and the latter are free oxygen ions that ensure charge neutrality. The complexity of this material lays in the different properties that can be imparted by changing the framework cations or the anion species that sit inside the cages. The free oxygen ions can be replaced by other anionic species such as $\mathrm{Cl}^{-}, \mathrm{F}^{-}, \mathrm{OH}^{-}, \mathrm{H}^{-}, \mathrm{NH}_{2}^{-}$, and electrons. ${ }^{14-18}$ Likewise, aliovalent and isovalent substitutions of the cation species lead to drastic changes in the characteristic properties of this material. ${ }^{19-23}$

Two main activation processes are known to render $\mathrm{Ca}_{12} \mathrm{Al}_{14} \mathrm{O}_{33}$ conductive: (i) hydrogen doping followed by ultraviolet (UV) light irradiation and (ii) removal of the free $\mathrm{O}^{2-}$ species through reduction by a base metal. ${ }^{3,24}$ While the former process transforms the insulator into a polaron conductor, the latter gives rise to free carrier metallic properties in the material.

Much has been said regarding the electronic structure of $\mathrm{Ca}_{12} \mathrm{Al}_{14} \mathrm{O}_{33}$ and the mechanisms ${ }^{25-27}$ that govern the transition from insulator to metal., ${ }^{9,28-30}$ However, this topic remains controversial mainly due to the intrinsic limitations of the theoretical models used to study transitions across a wide range of electron concentration.

Sushko et al., ${ }^{10}$ using embedded cluster and densityfunctional (DFT) approaches, suggested that a high degree of charge localization exists for low electron densities. However, for high electron densities their embedded cluster model diverges from the results of their plane-wave DFT showing high localization for the former and gradual delocalization for the latter. ${ }^{10}$ As $\mathrm{Li}$ et al. ${ }^{30-34}$ mentioned, the embedded cluster technique should only be used in the dilute limit of carrier concentration; thus, extrapolation of the results from the $\mathrm{H}$ doped UV-irradiated $\mathrm{Ca}_{12} \mathrm{Al}_{14} \mathrm{O}_{33}$ system to the $\left[\mathrm{Ca}_{24} \mathrm{Al}_{28} \mathrm{O}_{64}\right]^{4+}$ $+4 \mathrm{e}^{-}$should not be made based on this approach. ${ }^{30}$ For the case of high electron concentration, Medvedeva and Freeman ${ }^{28}$ predicted a change in conduction mechanism from hopping to free-electron-like behavior and presented a model with high degree of charge delocalization.

Received: January 23, 2015

Revised: March 24, 2015

Published: March 31, 2015 
Palacios et al. ${ }^{35}$ determined by synchrotron X-ray diffraction the structure of electron loaded mayenite, $\left[\mathrm{Ca}_{12} \mathrm{Al}_{14} \mathrm{O}_{32}\right]^{2+} \mathrm{O}_{1-\delta}^{2-} \mathrm{e}_{2 \delta}^{-}(0<\delta<1)$ below the critical electron concentration where the semiconductor-to-metallic transition occurs showing high electron localization for the mayenite sample with composition $\left[\mathrm{Ca}_{12} \mathrm{Al}_{14} \mathrm{O}_{32}\right]^{2+} \mathrm{O}_{0.55}^{2-} \mathrm{e}_{0.9}^{-}$.

Matsuishi et al. ${ }^{36}$ measured the optical properties of mayenites with conductivities ranging from 2 to $1500 \Omega^{-1}$ $\mathrm{cm}^{-1}$. They proposed that the coexistence of mobile (Drudelike) and localized carriers at all dopings suggests a strong electron-phonon interaction. However, the spectral range they measured does not go far enough into the infared to cover the response of all active phonons. In this work we analyze the optical conductivity of the metal-insulator transition driven by electron doping in mayenites. In particular, we measure the full infrared range where optically active phonons are expected. In the insulating samples all phonons are described by symmetric Lorentz oscillators. We find that when the material undergoes the metal-insulator transition, some of the phonons acquire an asymmetric Fano profile, indicative of a strong electronphonon coupling. This coupling increases with doping showing that free carriers favor the formation of polarons. We claim that these two excitations cooperate, rather than compete, in the formation of the conduction properties of mayenites. We also compare our results to band structure calculations stressing the differences between lightly and heavily doped samples.

\section{EXPERIMENTAL RESULTS}

Samples and Measurements. The samples for this work were prepared by the floating zone method. The growth was carried out in an optical image furnace (CSI FZ-T-10000-H-VIVP, Crystal Systems, Inc., Japan) equipped with four $1000 \mathrm{~W}$ tungsten halide lamps focused by four polished elliptical mirrors under flowing $\mathrm{N}_{2}$. In each case, the quality of the grown crystal was evaluated by X-ray diffraction and optical microscopy. After growth several samples were sliced from the main crystal and placed in sealed quartz tubes in the presence of Ti granules (99.99\%, Alfa Aesar). The quartz tubes containing the samples were placed in a tube furnace at $\sim 1000$ ${ }^{\circ} \mathrm{C}$ for various periods of time in order to obtain the range of samples shown in Figure 1. The specimens investigated here span the whole range from a transparent insulating sample $\left(10^{-10} \Omega^{-1} \mathrm{~cm}^{-1}\right)$ to a black metallic $\left(1500 \Omega^{-1} \mathrm{~cm}^{-1}\right)$ sample. These values for the dc conductivity were obtained by fourpoint-probe measurements after deposition of platinum contacts on the samples surfaces.

Raw Data. Near normal reflectance measurements were performed at room temperature in a Bruker IFS66v Fouriertransform spectrometer over the $2.5 \mathrm{meV}-2 \mathrm{eV}(20-16000$ $\mathrm{cm}^{-1}$ ) range, using gold and aluminum mirrors as references. They were extended to the ultraviolet, up to $7 \mathrm{eV}$ (55000 $\mathrm{cm}^{-1}$ ), in a Cary 5000 spectrometer. This instrument was also utilized to measure the transmission from 0.4 to $7 \mathrm{eV}$ (3300$\left.55000 \mathrm{~cm}^{-1}\right)$. The samples were mechanically polished and display a shiny surface. However, defects (bubbles and microcracks) are still present. All samples had both faces polished, and the reflectance was measured selecting the best mirror-like face.

Transmittance. Figure 1 shows the transmission spectra for the transparent samples $(1,2$, and 3$)$. The remaining metallic samples are too opaque to produce any usable signal. The transmission is heavily affected by the samples microstructure, and the ordinate units are not absolute. The actual average

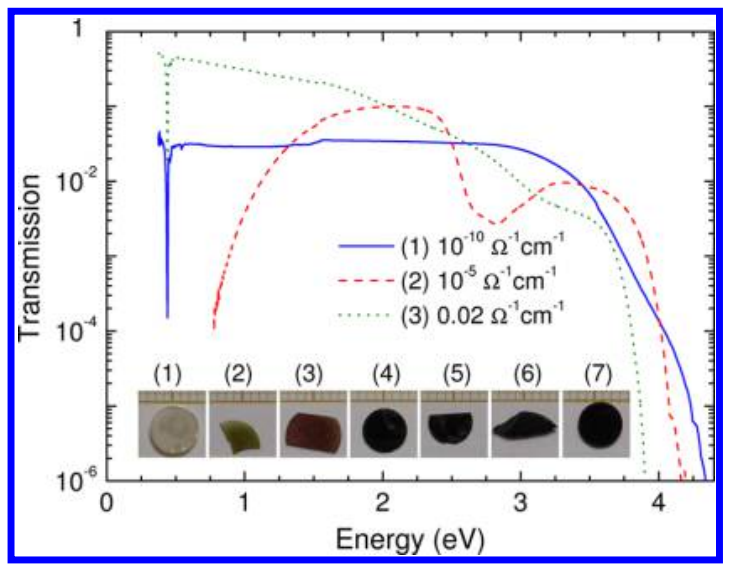

Figure 1. Transmission spectra of the three most insulating samples (1 to 3 ) in the visible and UV range. The pictures, from left to right, are samples 1 to 7 having dc conductivities of $10^{-10}, 10^{-5}, 0.02,15,120$, 200 , and $1500 \Omega^{-1} \mathrm{~cm}^{-1}$. The absolute level of the transmission is not realistic as the data are strongly affected by the bulk microstructure (cracks and bubbles). One should only consider peak positions in the above data.

transmittance should be higher than the value shown. On the basis of the reflectance, we estimate it to be in the 0.7-0.9 range. Note that sample 2 shows significant dispersion around 1 $\mathrm{eV}$, which suggests a large scattering. Indeed, visual inspection of this sample indicates the presence of numerous bubbles in the bulk and hence that the observed dispersion is not an intrinsic property of this material.

The transmittance shown in a log scale displays the same shape as the absorbance (with a sign change). We observe a line at $0.44 \mathrm{eV}$ in samples 1 and 3, likely due to water molecules adsorbed in the material. All samples show an abrupt onset of absorption around $3.5 \mathrm{eV}$, which corresponds to the band gap. Sample 1 has a constant transmission up to this energy scale, as expected from an insulator $\left(10^{-10} \Omega^{-1} \mathrm{~cm}^{-1}\right)$. Sample 3, which is much less insulating $\left(0.02 \Omega^{-1} \mathrm{~cm}^{-1}\right)$, displays an increasing absorption with frequency and a shoulder around $3.2 \mathrm{eV}$, just before the absorption edge at $\sim 3.5 \mathrm{eV}$. This suggests the presence of absorption above $\sim 2 \mathrm{eV}$, hence the reddish color of the sample. The black color of the metallic samples indicates that there is absorption spread over the whole visible range.

Reflectance. Opposite to the transmittance, the reflectance has little sensitivity to bulk sample defects. What matters the most is the quality of the surface. Figure 2 shows the reflectance for all samples. The main panel shows the spectrum up to the deep UV range $(6.5 \mathrm{eV})$.

Samples 1-3 show the typical reflectance for insulators. A set of sharp phonon features, shown in Figure 2a, dominates the far-infrared reflectance below $0.15 \mathrm{eV}$. The spectra become approximately constant up to $\sim 4 \mathrm{eV}$. A hint of an absorption peak is visible slightly above $6.5 \mathrm{eV}$, i.e., above the absorption onset seen at $\sim 3.5 \mathrm{eV}$ in Figure 1.

The insulating samples have a transparency window from approximately 0.3 to $4 \mathrm{eV}$. In this range, both the reflection of the back surface and the transmission through the bulk contribute to the total measured reflectance. In order to avoid extraneous features in Kramers-Kronig analysis (see Optical Conductivity section), we utilized the data of Figure 1 to account for these effects. In Figure $2 b$, we show the correction needed for sample 3, which required the largest correction. As a consequence of this correction, the absorption line at $0.4 \mathrm{eV}$ is 


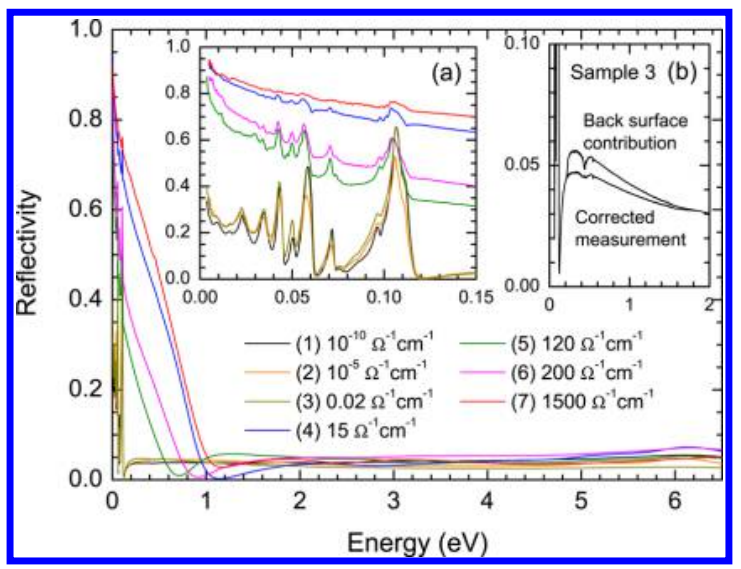

Figure 2. Main panel shows the reflectance of all seven samples studied in the full measured spectral range. Inset (a) displays the farinfrared reflectance showing phonons in the insulating samples and their gradual screening as the free-carriers response appears in metallic samples. Inset (b) shows the correction in the reflectance (due to a double bounce front and back surfaces reflections) needed for Kramers-Kronig. Only the insulating samples are concerned. The data shown are for sample 3 , where the correction was the largest.

strongly suppressed, and the overall reflectance level in the transparency window decreases by less than $\sim 1 \%$. Reflection of the back surface is also present in the very far-infrared and appears as a reflectance increase below $15 \mathrm{meV}$. This upturn in the reflectance should not be mistaken with a free-carrier response. It is an artifact due to the transparency of any insulating sample. For Kramers-Kronig purposes, we disregarded the data below $15 \mathrm{meV}$ for samples $1-3$. None of the above issues concern the opaque conducting samples.

Samples 4-7 exhibit a metallic behavior as indicated by the large enhancement of the reflectance below roughly $1 \mathrm{eV}$. Figure 2a shows the gradual screening of phonon peaks by the increasing reflectance due to free carriers in the far-infrared. This is a standard behavior in conducting oxides where doping drives the sample from insulating to metallic. For the most metallic samples (from the spectroscopic point of view those are samples 4 and 7 ), the reflectance approaches unity at low frequencies, as expected for a metal. A minimum develops at 0.7 and $0.9 \mathrm{eV}$ for samples 5 and 6 , respectively, and $\approx 1.1 \mathrm{eV}$ for samples 4 and 7. This minimum, in a simple Drude-like picture, is the plasma edge, whose frequency moves up as the electron concentration increases. It is important to remark that in sample 7 alone the reflectivity at this minimum is not close to zero, an indication that this sample does not follow a simple Drude behavior.

\section{OPTICAL CONDUCTIVITY}

We performed a Kramers-Kronig transform of the reflectance in order to extract the optical conductivity. ${ }^{37}$ To do so, the spectra must be extrapolated from the lowest available experimental frequency down to zero and from the highest available experimental frequency up to infinity.

In samples 4-7 we utilized a Hagen-Rubens $1-A \sqrt{ } \omega$ expression, where $A$ is a constant, that represents the reflectance of a Drude free electron gas in the low frequency limit. In the insulating samples $(1-3)$ we adopted a constant extrapolation. Utilizing other extrapolations does not affect the computed optical conductivity by more than $2 \%$ beyond twice the measured lowest frequency.
The high-energy extrapolation choice is a more sensitive matter. It was realized earlier that the high-frequency extrapolation biases significantly the optical conductivity over a range of the order of $1 / 3$ of the maximum available experimental frequency. ${ }^{38}$

In many oxides, such as cuprates, ${ }^{39}$ manganites, ${ }^{40}$ and nickelates, ${ }^{41}$ one can take advantage of available reflectance data of a parent compound measured at very high energies (30-36 $\mathrm{eV})$. In such a case, the resulting optical conductivity can be trusted up to 5-10 eV. Such high-energy data are not available for mayenites and the high-frequency extrapolation in our case is more delicate. A standard procedure is to select a cutoff frequency beyond which we ignore a possible contribution from very high energy bands and merely append a free electron $\omega^{-4}$ decay. We chose a $12 \mathrm{eV}$ cutoff, which produces similar visible-UV spectra for all samples. We also tried the method recently proposed by Tanner ${ }^{42}$ and found similar results.

The optical conductivity of all seven samples is shown in Figures 3 and 4 . We report, as solid circles, the independently

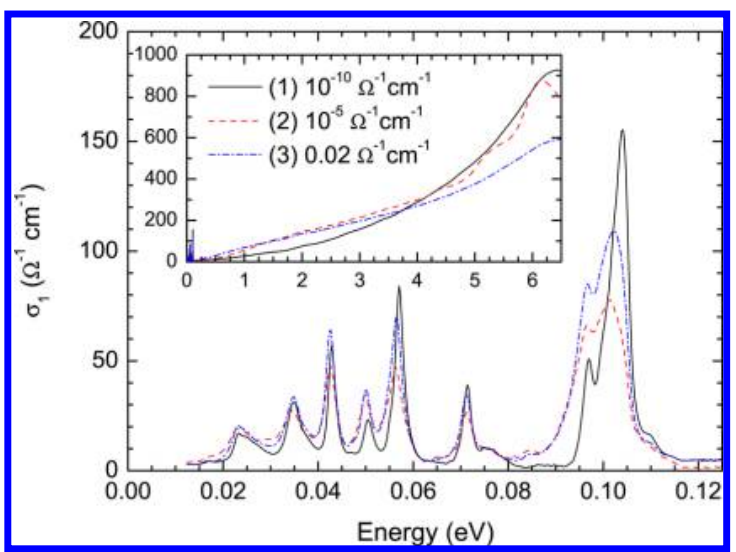

Figure 3. Real part of the optical conductivity for the three insulating samples. The far-infrared is dominated by phonon peaks. The inset shows the optical conductivity in the visible and UV regions.

measured dc conductivity for samples $4-7$. The zero energy extrapolation of the optical conductivity agrees well with the dc

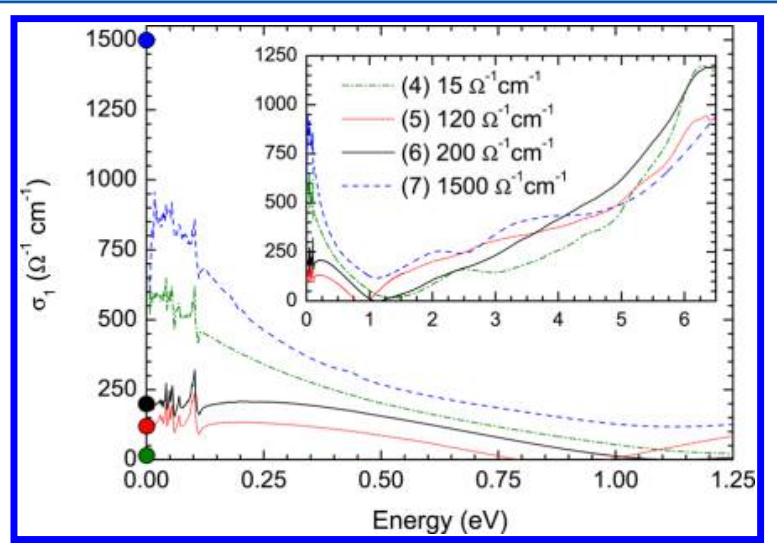

Figure 4. Real part of the optical conductivity for the four conducting samples. The main panel shows a Drude-like peak developing with increasing doping. The solid circles at zero energy are the measured dc conductivity. The inset shows the visible and UV portions of the spectra. The marked minimum around $1 \mathrm{eV}$ shows the boundary between interband transitions (above $1 \mathrm{eV}$ ) and the intraband mobile carriers response (below $1 \mathrm{eV}$ ). 
values, except for sample 4, which appears more metallic than expected from the dc data.

The optical conductivity for sample 5 becomes slightly negative $\left(-12 \Omega^{-1} \mathrm{~cm}^{-1}\right)$ in the $0.7-1 \mathrm{eV}$ range. This is by and large within our error bars, merely meaning that $\sigma_{1}(\omega)$ has a minimum close to zero as, indeed, displayed by the other metallic samples 4 and 6. Overall, the general trend when going from the most insulating to the most metallic sample is nicely replicated in the optical conductivity. Although phonons are more prominent in samples $1-3$, they remain visible as sharp structures in the metallic samples. We will comment on their line shapes in the next section.

All samples have been analyzed as described above. However, we will focus our discussion on samples 1,6 , and 7. Sample 1 is kept as being representative of all three (1,2, and 3) insulating samples. Sample 6 is kept as being also representative of sample 5. Sample 7 is the most conducting one and must be considered as an end point.

Sample 4 is a special case. Among the metallic samples, it displays a noticeable inconsistency between its dc resistivity and the zero frequency extrapolation of its optical conductivity. Optically sample 4 looks more metallic (higher reflectance and larger optical conductivity) than samples 5 and 6 , despite its lower dc conductivity. Although we cannot make a definite statement, this effect is, in general, related to resistive domain boundaries in the sample. Such boundaries decrease the electrically measured dc conductivity but go virtually unnoticed in the optical, contactless, measurement. The optically derived resistivity of sample 4 should be closer to $500 \Omega^{-1} \mathrm{~cm}^{-1}$. For the remaining of this paper we will assume that the optical values for this sample are more reliable than the dc transport. However, we will use the results on this sample only to complete the data set, and no strong conclusion based solely on its response is taken.

\section{DATA ANALYSIS}

Insulating Samples. Sample 1 has the characteristic optical response of an insulator, namely (i) a set of sharp phonon lines in the infrared range and (ii) a slowly increasing visible-UV absorption associated with the band gap. As shown in Figure 3, all the phonon lines are found below $120 \mathrm{meV}\left(1000 \mathrm{~cm}^{-1}\right)$. With 116 atoms per unit cell, we have not attempted to identify the relatively small number of vibration modes that we observed. Note that only the three highest frequency phonons appear to be slightly broadened and shifted in sample 3, whereas the eight low-frequency ones map exactly those from sample 1. This suggests that the changes in the higher energy phonons are due to doping rather than deterioration of crystal quality.

The real part of the optical conductivity was fitted with a series of $N$ Lorentz oscillators of the sort

$$
\sigma_{1}=\frac{2 \pi}{Z_{0}} \sum_{j=1}^{N} \frac{\Delta \varepsilon_{j} \Omega_{j}^{2} \gamma_{j} \omega^{2}}{\left(\Omega_{j}^{2}-\omega^{2}\right)^{2}+\gamma_{j}^{2} \omega^{2}}
$$

where $Z_{0}$ is the vacuum impedance and $\Delta \varepsilon_{j}, \Omega_{j j}$, and $\gamma_{j}$ are respectively the dielectric strength, resonance frequency, and line width of each $j$ th oscillator.

We utilized 11 Lorentzians to reproduce the phonon lines in the spectrum of sample 1, as shown in Figure 5a. It is rather remarkable that a simple Lorentz fitting describes the data so well. The Lorentz form (as opposed to a Gaussian peak) indicate a low level of disorder.

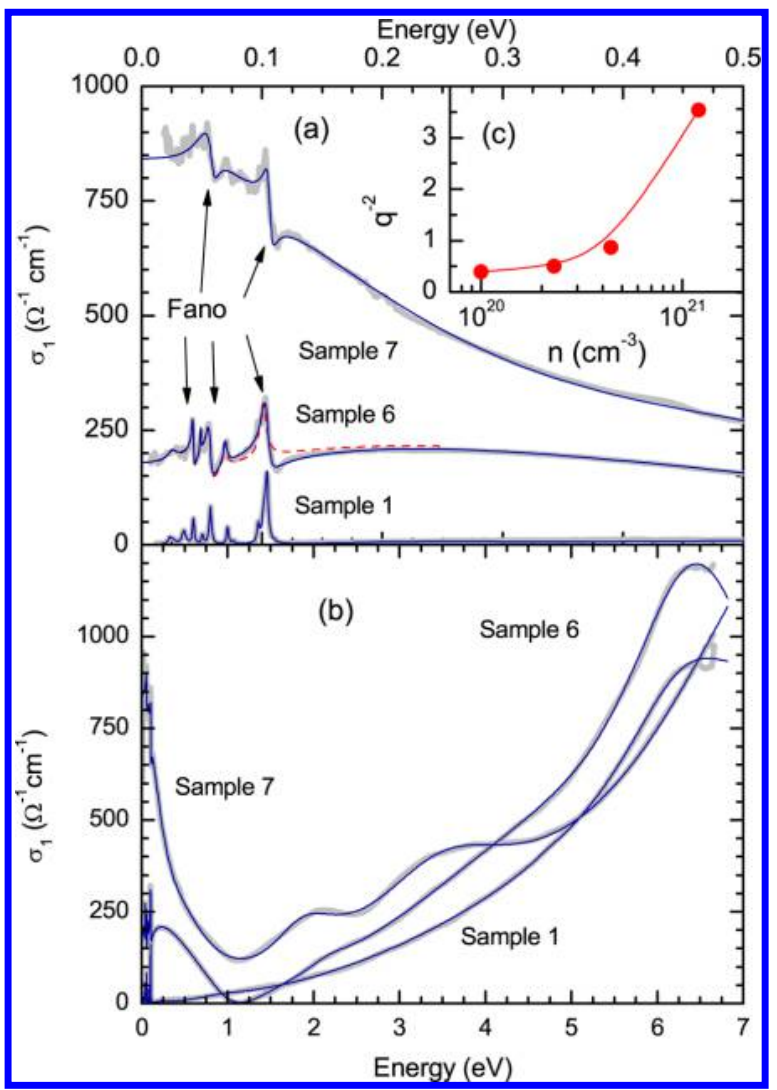

Figure 5. Panel (a) shows the optical conductivity for samples 1, 6, and 7 as thick gray lines. The thin lines are fits using a series of Lorentzians and, in the metallic samples, a Drude peak and Fano oscillators. The phonons showing Fano profiles are indicated by the arrows. The dashed red line for sample 6 shows an alternative fit where a Lorentzian oscillator substitutes the Fano profile for the highest energy phonon. Panel (b) shows the fit over the full spectral range for the same three samples. The inset (c) shows the value of the FanoBreit-Wigner parameter $q^{-2}$ for the phonon located at $\sim 450 \mathrm{~cm}^{-1}$ as a function of carrier concentration. The solid line is a guide for the eye.

The same kinds of oscillators were used to describe the optical conductivity in the visible and UV ranges. The fit for sample 1 is shown in Figure $5 \mathrm{~b}$. In the $1-5 \mathrm{eV}$ range, we notice that there is a large spectral weight that comes mostly from the tail of UV oscillators at and above $6 \mathrm{eV}$. In samples 2 and 3 (not shown in Figure 5), small oscillators appear in this $1-5 \mathrm{eV}$ range, accounting for the gradual color change, but they represent less than $10 \%$ of the spectral weight.

Metallic Samples. Figure 4 already shows that the optical conductivity of the metallic samples exhibits a qualitatively very different behavior due to the free electron contribution that emerges in the infrared. Below $1 \mathrm{eV}$ the optical conductivity is dominated by the response of conducting electrons, and above that energy, we find excitations within the gap below $6.5 \mathrm{eV}$. Figure 5a shows the far-infrared fits for samples 6 and 7 , including the sharp structures that correspond to the set of phonons identified in sample 1 .

The pure electronic contribution to the optical conductivity is best seen in Figure 5b. In sample 6 we notice a broad peak around $0.25 \mathrm{eV}$ followed by a minimum at $1 \mathrm{eV}$. In sample 7 , further development of the Drude contribution turns this feature into a shallow minimum, reminiscent of conducting oxides. $^{39-41}$ This $1 \mathrm{eV}$ minimum in the optical conductivity of the metallic samples corresponds to the crossover region 
between electronic transitions inside the conducting band (Drude term) and transitions between bands.

The free electron response is described by a simple Drude contribution:

$$
\sigma_{1}(\omega)=\frac{2 \pi}{Z_{0}} \frac{\Omega_{\mathrm{p}}{ }^{2} \tau}{1+\omega^{2} \tau^{2}}
$$

where $\Omega_{\mathrm{p}}$ is the plasma frequency and $\tau$ is the scattering time.

To fit the data in the high-energy $(>1 \mathrm{eV})$ region, we mimicked the increasing spectral weight-indicative of states below the UV gap-with Lorentz oscillators in the form of eq 1. Besides the large tail of the deep UV band gap transition, one can see structures in the $1-5 \mathrm{eV}$ range. They are more pronounced in sample 7 , but a close inspection of the data, as well as of the fitting parameters, shows that they are also present in sample 6 (and absent in the insulating sample 1).

In the far-infrared of the metallic samples, some phonons appear as asymmetric sharp structures (Figure 5c). Such an asymmetry may or may not be observed in oxides: there are asymmetric phonons in $\mathrm{Nd}_{2-x} \mathrm{Ce}_{x} \mathrm{CuO}_{4}$ at small $x,{ }^{43}$ but not in $\mathrm{La}_{2 / 3} \mathrm{Ca}_{1 / 3} \mathrm{MnO}_{3}$. ${ }^{44}$ The asymmetric shape is a signature of the Fano effect, which results from the coupling of well-defined excitations (phonons in our case) to an electronic continuum. ${ }^{45}$

In Fano's formalism, the initial discrete state, having energy $E_{\phi}$, is coupled to a set of states $\psi_{E}$ from the continuum producing the perturbed states $\Psi_{E}$. The ratio of the transition probability $\left|\left\langle\Psi_{E}|T| i\right\rangle\right|^{2}$ ( $T$ is the transition operator) to the unperturbed continuum $\left|\left\langle\psi_{E}|T| i\right\rangle\right|^{2}$ is given by the general equation

$$
\left|\frac{\left\langle\Psi_{E}|T| i\right\rangle}{\left\langle\psi_{E}|T| i\right\rangle}\right|^{2}=\frac{(q+\epsilon)^{2}}{1+\epsilon^{2}}
$$

where $q^{-2}$ is the Fano-Breit-Wigner parameter, which vanishes when the electron-phonon interaction disappears. $\epsilon$ is a reduced energy:

$$
\epsilon=\frac{E-E_{\phi}-F(E)}{\frac{1}{2} \gamma}
$$

$\gamma$ defines the energy spread over which the interaction occurs and includes the width of both the phonon and $q . F(E)$ is the line shift due to the coupling effect.

The optical conductivity relates directly (within a multiplying factor) to the transition probability. ${ }^{37}$ Although a Fano feature can be described simply by the product of eqs 2 and 3 , here we adopt the formalism proposed by Davis and Feldkamp, ${ }^{46}$ who generalized Fano's approach to multiple discrete states:

$$
\sigma_{1}^{\mathrm{F}}=\frac{2 \pi}{Z_{0}} R \omega\left\{\frac{[q \gamma+(\omega-\Omega)]^{2}}{\gamma^{2}+(\omega-\Omega)^{2}}-1\right\}
$$

where the new parameter $R$ is a renormalization factor that takes into account the transition rate between continuum and localized states.

We utilized Lorentz oscillators to fit the phonons in samples 6 and 7, whenever possible. For a few phonons, indicated by the arrows, a Fano profile was necessary. The dashed line for sample 6 in Figure 5a shows the effect of using a Lorentz term instead of a Fano profile to fit the highest energy phonon. The Lorentz oscillator alone cannot reproduce the pronounced minimum and the asymmetric shape of the phonon.
As observed by Matsuishi et al., ${ }^{36}$ the Drude term in the conducting samples coexists with localized Lorentz oscillators at all carrier concentrations. They suggested that this coexistence indicates the contribution of free carriers and polaronic hopping to the dc conductivity. Note that the mere presence of both excitations is not sufficient to substantiate such a claim. However, the presence of a strong Fano effect and, hence, strong electron-phonon coupling that we find in our analysis does provide a solid case for polaron formation. As we show in the inset $c$ of Figure 5, the Fano-Breit-Wigner parameter increases substantially with doping. The doping dependence of this parameter is shown for the phonon around $450 \mathrm{~cm}^{-1}$, but this dependence is qualitatively the same for the other two phonons - at 340 and $840 \mathrm{~cm}^{-1}$ - showing a strong Fano profile. The behavior of $q^{-2}$ indicates not only that do polarons and free carriers coexist but also that increasing carrier concentration favors the formation of polarons. Our results indicate that free carriers and polaronic hopping conduction are cooperative mechanisms rather than competing.

\section{ELECTRONIC BAND STRUCTURE CALCULATIONS}

We calculated the electronic band structure of stoichiometric $\mathrm{Ca}_{12} \mathrm{Al}_{14} \mathrm{O}_{33}$ and oxygen-deficient $\mathrm{Ca}_{12} \mathrm{Al}_{14} \mathrm{O}_{32}$ using the highly precise all-electron full-potential linearized augmented planewave (FLAPW) method. ${ }^{47,48}$ Within the local density approximation (LDA), we chose cutoffs of 17.64 Ry for the basis functions and $100 \mathrm{Ry}$ for the potential representation. An expansion in terms of spherical harmonics with $l \leq 8$ inside the muffin-tin spheres were used. Summations over the Brillouin zone were carried out using at least 20 special $\mathbf{k}$ points in the irreducible wedge for the self-consistent calculations and with at least 200 special $\mathbf{k}$ points for the optical properties (dielectric functions) calculations. For both structures, the internal positions of all atoms in the unit cell were optimized via the total energy and atomic forces minimization. The lattice parameter was kept at the experimental value.

We stress here that $\mathrm{Ca}_{12} \mathrm{Al}_{14} \mathrm{O}_{32}$ represents the case when all encaged oxygen ions are removed from the crystal and corresponds to an extra electronic concentration $n=2.33 \times$ $10^{21} \mathrm{~cm}^{-3}$. This is almost twice the estimated electron concentration for sample 7 , as discussed below.

To study the optical properties of insulating and metallic mayenite, we calculate the dielectric function, $\epsilon(\omega)=\epsilon_{1}(\omega)+$ $i \epsilon_{2}(\omega)$, within FLAPW. The imaginary part, $\epsilon_{2}(\omega)$, is related to the optical absorption at a given frequency $\omega$ and, in the long wavelength limit, is given by ${ }^{49}$

$$
\epsilon(\omega)=\frac{8 \pi^{2} e^{2}}{\omega^{2} m^{2} V} \sum_{c, v} \sum_{\mathbf{k}}|\langle c, \mathbf{k}|\hat{e} \cdot \mathbf{p}| v, \mathbf{k}\rangle|^{2} \delta\left[E_{c}(\mathbf{k})-E_{v}(\mathbf{k})-\hbar \omega\right]
$$

where the summation runs over all the conduction $(c)$ and valence $(v)$ states with momentum $\mathbf{k}$ and energies $E_{c}$ and $E_{v}$. $\hat{\mathbf{e}}$ and $\mathbf{p}$ are the polarization and momentum operators, respectively.

Figure 6 shows the electronic band structures of $\mathrm{Ca}_{12} \mathrm{Al}_{14} \mathrm{O}_{33}$ and $\mathrm{Ca}_{12} \mathrm{Al}_{14} \mathrm{O}_{32}$ calculated along the high-symmetry lines of the cubic Brillouin zone. The cage-like structure of mayenite results in the so-called cage (or cavity) conduction band (CCB) which, for $\mathrm{Ca}_{12} \mathrm{Al}_{14} \mathrm{O}_{33}$, consists of five bands associated with the five empty cages in the unit cell. The sixth cage is filled with $\mathrm{O}^{2-}$, giving rise to the fully occupied flat bands $\left(\mathrm{p}_{x}, \mathrm{p}_{y}, \mathrm{p}_{z}\right)$ below the Fermi level (Figure 6a). Because of the presence of 


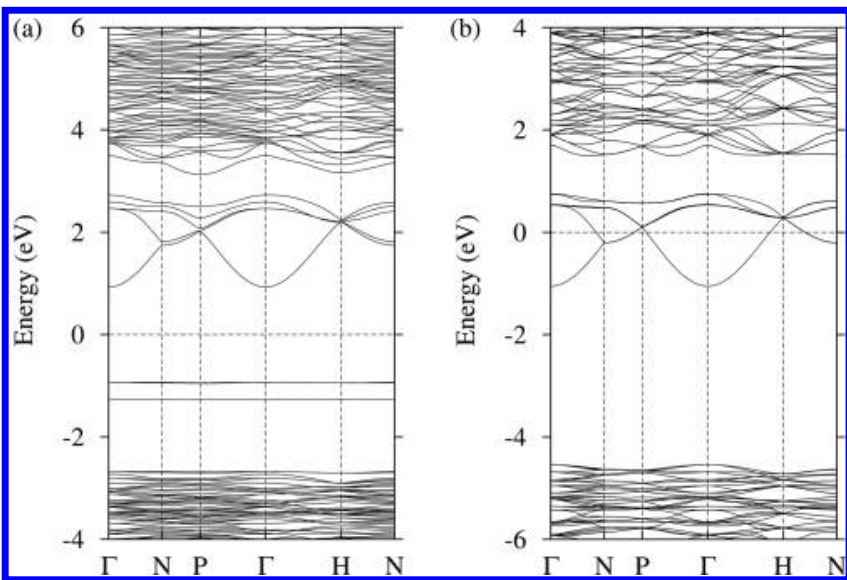

Figure 6. Electronic band structure of (a) stoichiometric and (b) oxygen-deficient $12 \mathrm{CaO} \cdot 7 \mathrm{Al}_{2} \mathrm{O}_{3}$. The encaged loosely bound $\mathrm{O}^{2-}$ ions give rise to the occupied flat bands below the Fermi level in (a). When these $\mathrm{O}^{2-}$ are removed, shown in (b), the Fermi level shifts up into the cage conduction band, giving rise to a metallic state.

the encaged oxygen ions, the CCB shifts to lower energies and sits well below the framework conduction band. Removal of the encaged $\mathrm{O}^{2-}$ ion leaves two extra electrons in the crystal. The cage conduction band of $\mathrm{Ca}_{12} \mathrm{Al}_{14} \mathrm{O}_{32}$ consists of six bands, shown in Figure $6 \mathrm{~b}$, associated with the six empty cages in a single unit cell. Because of the uniform charge distribution throughout all cages, ${ }^{28}$ the lowest conduction state does not split off when the oxygen vacancy is created.

Figures 7a,b show the calculated imaginary part of the dielectric function. Relating to Figure 6, we can identify the absorption peaks for both dopings.

Stoichiometric $\mathrm{Ca}_{12} \mathrm{Al}_{14} \mathrm{O}_{33}$. (i) Broad low-intensity peak A: electronic transitions from $\mathrm{p}_{x}, \mathrm{p}_{y}$, and $\mathrm{p}_{z}$ states of the encaged $\mathrm{O}^{2-}$ to the empty states of CCB. (ii) Peak B:

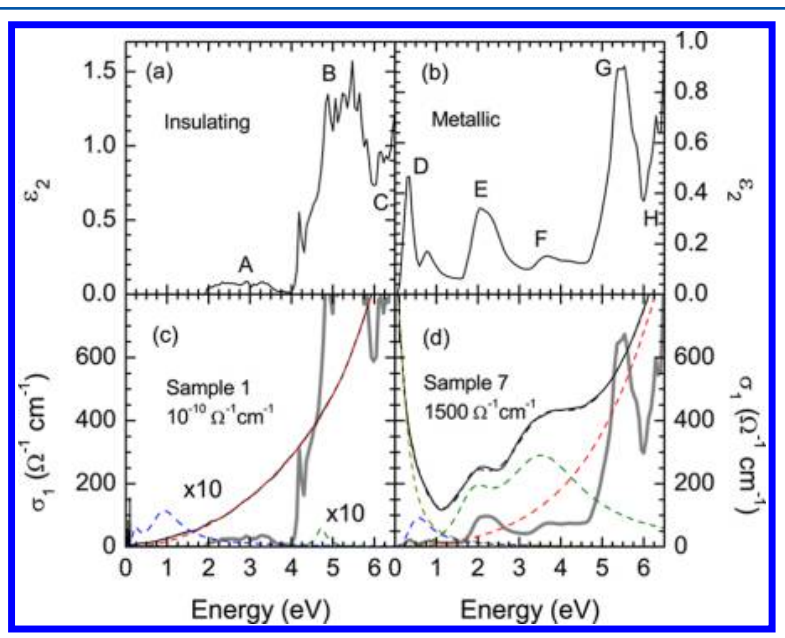

Figure 7. Calculated imaginary part of the dielectric function for (a) almost insulating mayenites with the cages filled and (b) empty cages conducting materials. The experimentally determined optical conductivity and fit are shown for (c) insulating sample 1 and (d) conducting sample 7. The fits are decomposed into the dashed lines. In the insulating samples the optical response is dominated by a tail due to absorptions above $5 \mathrm{eV}$. No Drude peak is present. The conducting sample has a zero frequency centered Drude peak and a distribution of Lorentz peaks from 1 to $5 \mathrm{eV}$. The thick gray lines correspond to $\sigma_{1}$ calculated from the values of $\varepsilon_{2}$ from (a) and (b). transitions from the valence band to CCB. (iii) Peak C: onset of intense transitions from the valence band to the framework conduction band.

Oxygen-Deficient $\mathrm{Ca}_{12} \mathrm{Al}_{14} \mathrm{O}_{32}$. (i) Double-peak D: electronic transitions within the partially filled CCB. The double-peak structure comes from the splitting of the upper two empty states at the $\Gamma$ point and along the [110] and [111] crystallographic directions that gives rise to a two-peak structure in the density of states just above the Fermi level. (ii) Peaks E and F: electronic transitions from the partially filled CCB to the framework conduction bands. The latter is composed primarily from the $\mathrm{Ca} d$ states. The crystal field splits these empty $\mathrm{d}$ states leading to two absorption peaks. (iii) Peaks $\mathrm{G}$ and $\mathrm{H}$ : transitions from the valence band to the $\mathrm{CCB}$ and framework conduction band, respectively.

In the next section, we compare our theoretical results with the experimental observations.

\section{DISCUSSION}

One can estimate the carrier density from the Drude term. Sample 7 has the largest Drude term with a plasma frequency $\Omega_{\mathrm{p}}=10500 \mathrm{~cm}^{-1}$. The plasma frequency is related to the carrier concentration $n$ by $\Omega_{\mathrm{p}}{ }^{2}=n e^{2} / \varepsilon_{0} m$, where $e$ and $m$ are the electronic charge and mass, respectively, and $\varepsilon_{0}$ is the vacuum permitivitty. In a first approximation we can take $m$ as the bare electron mass and we find $n=1.2 \times 10^{21} \mathrm{~cm}^{-3}$. Doing the same calculation for the remaining conducting samples, we obtain $n=$ $1.0 \times 10^{20} \mathrm{~cm}^{-3}$ for sample $5, n=2.3 \times 10^{20} \mathrm{~cm}^{-3}$ for sample 6 , and $n=4.4 \times 10^{20} \mathrm{~cm}^{-3}$ for sample 4 . These values are in the expected range for our samples. 50

We can also compare our spectra with $a b$ initio calculations. As the fine details of the doping evolution are hard to model and not very clear to extract from the data, we look here at the two extreme carrier concentrations, the insulating sample 1 and the highly conductive sample 7 . Figure 7 a shows the imaginary part of the dielectric function calculated for a system that represents sample 1 . Figure $7 \mathrm{~b}$ shows the same quantity for the extreme conducting limit-note that the extra electron in the calculations leads to $n=2.33 \times 10^{21} \mathrm{~cm}^{-3}$, twice the value estimated for sample 7 . Figures $7 \mathrm{c}, \mathrm{d}$ show the data and fits for samples 1 and 7. The dashed lines are individual Lorentz contributions divided up into four groups: (i) infrared excitations below $1 \mathrm{eV}$, (ii) visible-UV peaks between 1 and $5 \mathrm{eV}$; (iii) deep-UV terms above $5 \mathrm{eV}$; and (iv) the freeelectron zero-frequency-centered Drude contribution. The thick gray lines in Figures $7 \mathrm{c}$ and $7 \mathrm{~d}$ are the optical conductivity derived from the calculations of panels $\mathrm{a}$ and $\mathrm{b}$ through $\sigma_{1}=$ $2 \pi \omega \varepsilon_{2} / Z_{0}$.

The optical conductivity of sample 7 has a large oscillator around $6 \mathrm{eV}$ whose tail extends into the visible-UV range and accounts for some of the observed spectral weight. Nevertheless, most of the spectral weight in the visible-UV region comes, in fact, from two strong peaks at around 2 and $4 \mathrm{eV}$. Below $1 \mathrm{eV}$, the Drude peak dominates, but an extra Lorentz term is required at $\sim 0.5 \mathrm{eV}$ to properly describe the shape of $\sigma_{1}$. Our optical calculations find the three peaks around $0.5,2$, and $4 \mathrm{eV}$ observed in the conducting sample. However, their spectral weights are underestimated for the infrared and visible-UV peaks. This may be associated with the high symmetry of our conductive mayenite structure where all encaged $\mathrm{O}^{2-}$ were removed, resulting in an overall lattice relaxation and hence affecting the relative intensities of the optical transitions. 
We have to note that, as we mentioned in the Reflectance section, the reflectivity of sample 7 is not that of a simple Drude metal. The nonvanishing reflectivity around $1 \mathrm{eV}$ also leads to a large residual optical conductivity at $1 \mathrm{eV}$-compare $\sigma_{1}$ for samples 6 and 7 in Figure 5b. This effect is often related to a frequency-dependent scattering rate originated from electronelectron interactions. In our Drude-Lorentz model, part of the infrared Lorentz peak is actually used to account for this correction in the Drude term. This is a well-known situation in high-temperature superconducting cuprates, where one can always describe the infrared contribution due to free carriers by a Drude term and a so-called mid-infrared band. ${ }^{51}$

Note that the differences in the calculated and the observed absorption spectra may, in part, result from the fact that in the simulations perfect crystalline structures were studied so that the calculated peaks are more pronounced. However, the most important point is that the theoretically modeled metallic structure corresponds to all encaged oxygen ions being removed, which is not exactly the case for sample 7. An oxygen ion inside a cage makes the framework $\mathrm{Ca}$ atoms nonequivalent by symmetry. As a result, the two Ca types (two $\mathrm{Cal}$ atoms nearest to $\mathrm{O}^{2-}$ at a distance of $2.13 \AA$ from it and four $\mathrm{Ca} 2$ atoms farther away from the free oxygen, at $3.68 \AA$ ) give different contributions to the density of states in the framework conduction band. Namely, the $d$ states of the $\mathrm{Ca} 2$ atoms are energetically further away from the cage conduction band. This may result in the redistribution of the spectral weights of the 2 and $4 \mathrm{eV}$ peaks, with peak $\mathrm{F}$ being higher as compared to peak E (Figure $7 \mathrm{~b}$ ). Furthermore, the location of the cage conduction band with respect to the valence band and the framework conduction band is sensitive to the variations in the lattice parameter and in the atomic positions. As a result, peaks $B$ and $G$ are structure-sensitive and, hence, may not be resolved on experiment.

Moving to the insulating sample 1, where the Drude peak has disappeared, one can see that almost all of the visible-UV spectral weight comes, in fact, from the tail of the Lorentz term used to describe the large $6 \mathrm{eV}$ band gap absorption. Two very small peaks are present at 1 and $5 \mathrm{eV}$, but no other excitations exist in between. The measured transmission and optical conductivity of sample 1 show a broad absorption edge with an onset at $3 \mathrm{eV}$, extending up to $4.3 \mathrm{eV}$. The optical conductivity at $3 \mathrm{eV}$ is about $10 \%$ of its value at $7 \mathrm{eV}$, and this is indeed what is seen in the calculated optical conductivity, which has a very small spectral weight below $4 \mathrm{eV}$.

The conducting materials show phonons with asymmetric Fano line shapes. Although an electron-phonon interaction does not imply a Fano profile for the phonons, the converse is generally true. The fact that we find this coupling between the conducting electronic background and some phonons supports the presence of polaronic excitations in the material. As a final remark, the observed electron-phonon interaction should be considered as one possible realistic mechanism for the lowtemperature superconductivity of the metallic mayenite. ${ }^{8}$

\section{CONCLUSION}

We measured the reflectance of $\mathrm{Ca}_{12} \mathrm{Al}_{14} \mathrm{O}_{33}$ undergoing a metal-insulator transition as a function of thermal treatment. The optical conductivity calculated from the reflectance shows clear trends: (i) the insulator-metal transition induces the appearance of a Drude-like peak in the infrared whose weight is compatible with the charge densities expected for these materials; (ii) going from insulator to metal is accompanied by a spectral weight increase in the visible region; (iii) the appearance of a metallic state induces a Fano profile for phonons. All the samples show spectral weight throughout the whole spectral range, but strong peaks in the visible-UV region are present only in the conducting samples. This visible-UV optical conductivity agrees with ab initio calculated optical functions, which provides further insight into the origin of the observed transitions. The presence of Fano features in the metallic samples stresses the existence of a strong electronphonon coupling, which could possibly yield the observed lowtemperature superconductivity. As the Fano-Breit-Wigner parameter increases substantially with carrier concentration, we conclude that free carriers are not competing with polarons. These two excitations cooperate in the construction of the material conductivity.

\section{AUTHOR INFORMATION}

\section{Corresponding Author}

*Phone +33 (0)1 407944 85; e-mail lobo@espci.fr (R.P.S.M.L.).

\section{Present Address}

M.I.B.: Arizona State University, Tempe, AZ 85287.

Notes

The authors declare no competing financial interest.

\section{ACKNOWLEDGMENTS}

This work was supported by the MRSEC program of the National Science Foundation at Northwestern University (DMR-1121262) and by the Department of Energy (DEFG02-88ER45372).

\section{REFERENCES}

(1) Lacerda, M.; Irvine, J. T. S.; Glasser, F. P.; West, A. R. High Oxide Ion Conductivity in $\mathrm{Ca}_{12} \mathrm{Al}_{14} \mathrm{O}_{33}$. Nature 1988, 332, 525-526.

(2) Irvine, J. T. S.; Lacerda, M.; West, A. R. Oxide Ion Conductivity in $\mathrm{Ca}_{12} \mathrm{Al}_{14} \mathrm{O}_{33}$. Mater. Res. Bull. 1988, 23, 1033-1038.

(3) Hayashi, K.; Matsuishi, S.; Kamiya, T.; Hirano, M.; Hosono, H. Light-Induced Conversion of an Insulating Refractory Oxide into a Persistent Electronic Conductor. Nature 2002, 419, 462-465.

(4) Medvedeva, J. E.; Freeman, A. J. Combining High Conductivity with Complete Optical Transparency: A Band Structure Approach. Europhys. Lett. 2005, 69, 583-587.

(5) Hosono, H.; Hayashi, K.; Hirano, M. Active Anion Manipulation for Emergence of Active Functions in the Nanoporous Crystal $12 \mathrm{CaO}$. $7 \mathrm{Al}_{2} \mathrm{O}_{3}:$ A Case Study of Abundant Element Strategy. J. Mater. Sci. 2007, 40, 1872-1883.

(6) Fujita, S.; Ohkawa, M.; Suzuki, K.; Shibasaki, Y.; Mori, T. Reaction of Hydrogrossular with Hydrogen Chloride Gas at High Temperature. Chem. Mater. 2001, 13, 2523.

(7) Toda, Y.; Miyakawa, M.; Hayashi, K.; Kamiya, T.; Hirano, M.; Hosono, H. Field Emission of Electron Anions Clathrated in Subnanometer-Sized Cages in $\left[\mathrm{Ca}_{24} \mathrm{Al}_{28} \mathrm{O}_{64}\right]^{4+}\left(4 \mathrm{e}^{-}\right) . A d v$. Mater. 2004, 16, 685.

(8) Miyakawa, M.; Kim, S.; Hirano, M.; Kohama, S.; Kawaji, H. Superconductivity in an Inorganic Electride $12 \mathrm{CaO} \cdot 7 \mathrm{Al}_{2} \mathrm{O}_{3}$ :e. J. Am. Chem. Soc. 2007, 129, 7270-7271.

(9) Sushko, P. V.; Shluger, A. L.; Hirano, M.; Hosono, H. InsulatorConductor Transition in $12 \mathrm{CaO} \cdot 7 \mathrm{Al}_{2} \mathrm{O}_{3}$ Films: On the Stability of the Crystal Lattice under $\mathrm{Ar}^{+}$Bombardment. Thin Solid Films 2008, 516, 1350-1353.

(10) Sushko, P.; Shluger, A.; Hirano, M.; Hosono, H. From Insulator to Electride: A Theoretical Model of Nanoporous Oxide $12 \mathrm{CaO}$. $7 \mathrm{Al}_{2} \mathrm{O}_{3}$. J. Am. Chem. Soc. 2007, 129, 942-951.

(11) Li, J.; Yin, B.; Fuchigami, T.; Inagi, S.; Hosono, H.; Ito, S. Application of $12 \mathrm{CaO} \cdot 7 \mathrm{Al}_{2} \mathrm{O}_{3}$ Electride as a New Electrode for 
Superoxide Ion Generation and Hydroxylation of an Arylboronic Acid. Electrochem. Commun. 2012, 17, 52-55.

(12) Yoshizumi, T.; Hayashi, K. Thermionic Electron Emission from a Mayenite Electride Metallic Titanium Composite Cathode. Appl. Phys. Express 2013, 6, 015802.

(13) Bartl, H.; Scheller, T. Zur Struktur des $12 \mathrm{CaO} \cdot 7 \mathrm{Al}_{2} \mathrm{O}_{3}$. Neues Jahrb. Mineral., Monatsh. 1970, 35, 547-552.

(14) Hayashi, K.; Muramatsu, H.; Matsuishi, S.; Kamiya, T.; Hosono, $\mathrm{H}$. Humidity-Sensitive Electrical Conductivity in $\mathrm{Ca}_{12} \mathrm{Al}_{14-\mathrm{x}} \mathrm{Si}_{\mathrm{x}} \mathrm{O}_{32} \mathrm{Cl}_{2+\mathrm{x}}$ $(0<\mathrm{x}<3.4)$ Ceramics. Electrochem. Solid State Lett. 2009, 12, J11J13.

(15) Hayashi, K.; Sushko, P.; Ramo, D.; Shluger, A.; Watauchi, S.; Tanaka, I.; Matsuishi, S.; Hirano, M.; Hosono, H. Nanoporous Crystal $12 \mathrm{CaO} \cdot 7 \mathrm{Al}_{2} \mathrm{O}_{3}$ : A Playground for Studies of Ultraviolet Optical Absorption of Negative Ions. J. Phys. Chem. B 2007, 111, 1946-1956. (16) Polfus, J.; Toyoura, K.; Hervoches, C.; Sunding, M.; Tanaka, I.; Haugsrud, R. Nitrogen and Hydrogen Defect Equilibria in $\mathrm{Ca}_{12} \mathrm{Al}_{14} \mathrm{O}_{33}$ : A Combined Experimental and Computational Study. J. Mater. Chem. 2012, 22, 15828-15835.

(17) Strandbakke, R.; Kongshaug, C.; Haugsrud, R.; Norby, T. HighTemperature Hydration and Conductivity of Mayenite, $\mathrm{Ca}_{12} \mathrm{Al}_{14} \mathrm{O}_{33}$. J. Phys. Chem. C 2009, 113, 8938-8944.

(18) Hayashi, K. Heavy Doping of $\mathrm{H}^{-}$ion in $12 \mathrm{CaO} \cdot 7 \mathrm{Al}_{2} \mathrm{O}_{3}$. J. Solid State Chem. 2011, 184, 1428-1432.

(19) Bertoni, M. I.; Mason, T. O.; Medvedeva, J. E.; Freeman, A. J.; Poeppelmeier, K. R.; Delley, B. Tunable Conductivity and Conduction Mechanism in an Ultraviolet Light Activated Electronic Conductor. J. Appl. Phys. 2005, 97, 103713.

(20) Bertoni, M. I.; Mason, T. O.; Medvedeva, J. E.; Freeman, A. J.; Poeppelmeier, K. R. Enhanced Electronic Conductivity in SiSubstituted Calcium Aluminate. J. Appl. Phys. 2007, 102, 113704.

(21) Palacios, L.; Bruque, S.; Aranda, M. Structure of Gallium-Doped Mayenite and Its Reduction Behaviour. Phys. Status Solidi B 2008, 245, $666-672$.

(22) Maurelli, S.; Ruszak, S.; Witkowski, M.; Pietrzyk, P.; Chiesa, M.; Sojka, Z. Spectroscopic CW-EPR and HYSCORE Investigations of $\mathrm{Cu}^{2+}$ and $\mathrm{O}^{2-}$ Species in Copper Doped Nanoporous Calcium Aluminate $\left(12 \mathrm{CaO} \cdot 7 \mathrm{Al}_{2} \mathrm{O}_{3}\right.$. Phys. Chem. Chem. Phys. 2010, 12, 1093310941.

(23) Hayashi, K.; Ueda, N.; Matsuishi, S.; Hirano, M.; Kamiya, T.; Hosono, H. Solid State Syntheses of $12 \mathrm{SrO}^{-} 7 \mathrm{Al}_{2} \mathrm{O}_{3}$ and Formation of High Density Oxygen Radical Anions, $\mathrm{O}^{-}$and $\mathrm{O}_{2}^{-}$. Chem. Mater. 2008, 20, 5987-5996.

(24) Matsuishi, S.; Toda, Y.; Miyakawa, M.; Hayashi, K.; Kamiya, T.; Hirano, M.; Tanaka, I.; Hosono, H. High-Density Electron Anions in a Nanoporous Single Crystal: $\left[\mathrm{Ca}_{24} \mathrm{Al}_{28} \mathrm{O}_{64}\right]^{4+}\left(4 \mathrm{e}^{-}\right)$. Science 2003, 301, 626-629.

(25) Janek, J.; Lee, D. Defect Chemistry of the Mixed Conducting Cage Compound $\mathrm{Ca}_{12} \mathrm{Al}_{14} \mathrm{O}_{33}$. J. Korean Ceram. Soc. 2010, 47, 99105.

(26) Hosono, H.; Hayashi, K.; Kajihara, K.; Sushko, P. V.; Shluger, A. L. Oxygen Ion Conduction in $12 \mathrm{CaO} \cdot 7 \mathrm{Al}_{2} \mathrm{O}_{3}: \mathrm{O}^{2-}$ Conduction Mechanism and Possibility of $\mathrm{O}^{-}$Fast Conduction. Solid State Ionics 2009, 180, 550-555.

(27) Shluger, A. L.; McKenna, K. P.; Sushko, P. V.; Ramo, D. M.; Kimmel, A. V. Modelling of Electron and Hole Trapping in Oxides. Model. Simul. Mater. Sci. Eng. 2009, 17, 084004.

(28) Medvedeva, J. E.; Freeman, A. J. Hopping Versus Bulk Conductivity in Transparent Oxides: $12 \mathrm{CaO} \cdot 7 \mathrm{Al}_{2} \mathrm{O}_{3}$. Appl. Phys. Lett. 2004, 85, 955-957.

(29) Medvedeva, J. E.; Freeman, A. J.; Bertoni, M. I.; Mason, T. O. Electronic Structure and Light-Induced Conductivity of a Transparent Refractory Oxide. Phys. Rev. Lett. 2004, 93, 016408.

(30) Li, Z. Y.; Yang, J. L.; Hou, J. G.; Zhu, Q. S. Is Mayenite Without Clathrated Oxygen an Inorganic Electride? Angew. Chem., Int. Ed. 2004, 43, 6479-6482.

(31) Li, Z. Y.; Yang, J. L.; Hou, J. G.; Zhu, Q. S. Inorganic Electrides. Chem.-Eur. J. 2004, 10, 1592-1596.
(32) Li, Z. Y.; Yang, J. L.; Hou, J. G.; Zhu, Q. S. Inorganic Electride: Theoretical Study on Structural and Electronic Properties. J. Am. Chem. Soc. 2003, 125, 6050-6051.

(33) Li, Z. Y.; Yang, J. L.; Hou, J. G.; Zhu, Q. S. A First-Principles Study on Quasi-1D Alkali Metal Chains within Zeolite Channels. J. Chem. Phys. 2004, 120, 9725-9728.

(34) Li, H.; Mahanti, S. D. Theoretical Study of Encapsulated Alkali Metal Atoms in Nanoporous Channels of ITQ-4 Zeolite: OneDimensional Metals and Inorganic Electrides. Phys. Rev. Lett. 2004, 93, 216406.

(35) Palacios, L.; Cabeza, A.; Bruque, S.; Garcia-Granda, S.; Aranda, M. Structure and Electrons in Mayenite Electrides. Inorg. Chem. 2008, 47, 2661-2667.

(36) Matsuishi, S.; Kim, S. W.; Kamiya, T.; Hirano, M.; Hosono, H. Localized and Delocalized Electrons in Room-Temperature Stable Electride $\left[\mathrm{Ca}_{24} \mathrm{Al}_{28} \mathrm{O}_{64}\right]^{4+}\left(\mathrm{O}^{2-}\right)_{2-\mathrm{x}}\left(\mathrm{e}^{-}\right)_{2 \mathrm{x}}$ : Analysis of Optical Reflectance Spectra. J. Phys. Chem. C 2008, 112, 4753.

(37) Wooten, F. Optical Properties of Solids; Academic Press: New York, 1972.

(38) Santander-Syro, A. F.; Lobo, R. P. S. M.; Bontemps, N.; Lopera, W.; Girata, D.; Konstantinovic, Z.; Li, Z. Z.; Raffy, H. In-plane Electrodynamics of the Superconductivity in $\mathrm{Bi}_{2} \mathrm{Sr}_{2} \mathrm{CaCu}_{2} \mathrm{O}_{8+\delta}$ : Energy Scales and Spectral Weight Distribution. Phys. Rev. B 2004, 70, 134504.

(39) Uchida, S.; Ido, T.; Takagi, H.; Arima, T.; Tokura, Y.; Tajima, S. Optical-Spectra of $\mathrm{La}_{2-\mathrm{x}} \mathrm{Sr}_{\mathrm{x}} \mathrm{CuO}_{4}$ - Effect of Carrier Dpping on the Electronic Structure of the $\mathrm{CuO}_{2}$ Plane. Phys. Rev. B 1991, 43, 79427954.

(40) Okimoto, Y.; Katsufuji, T.; Ishikawa, T.; Urushibara, A.; Arima, T.; Tokura, Y. Anomalous Variation of Optical-Spectra With Spin Polarization in Double-Exchange Ferromagnet - $\mathrm{La}_{1-\mathrm{x}} \mathrm{Sr}_{\mathrm{x}} \mathrm{MnO}_{3}$. Phys. Rev. Lett. 1995, 75, 109-112.

(41) Katsufuji, T.; Okimoto, Y.; Arima, T.; Tokura, Y.; Torrance, J. B. Optical Spectroscopy of the Metal-Insulator-Transition in $\mathrm{NdNiO}_{3}$. Phys. Rev. B 1995, 51, 4830-4835.

(42) Tanner, D. B. Use of X-Ray Scattering Functions in KramersKronig Analysis of Reflectance. Phys. Rev. B 2015, 91, 035123.

(43) Lupi, S.; Capizzi, M.; Calvani, P.; Ruzicka, B.; Maselli, P.; Dore, P.; Paolone, A. Fano Effect in the a-b Plane of $\mathrm{Nd}_{1.96} \mathrm{Ce}_{0.04} \mathrm{CuO}_{4+y}$ : Evidence of Phonon Interaction with a Polaronic Background. Phys. Rev. B 1998, 57, 1248-1252.

(44) Boris, A. V.; Kovaleva, N. N.; Bazhenov, A. V.; van Bentum, P. J. M.; Rasing, T.; Cheong, S. W.; Samoilov, A. V.; Yeh, N. C. Infrared Studies of a $\mathrm{La}_{0.67} \mathrm{Ca}_{0.33} \mathrm{MnO}_{3}$ Single Crystal: Optical Magnetoconductivity in a Half-Metallic Ferromagnet. Phys. Rev. B 1999, 59, R697-R700.

(45) Fano, U. Effects of Configuration Interaction on Intensities and Phase Shifts. Phys. Rev. 1961, 124, 1866-1878.

(46) Davis, L. C.; Feldkamp, L. A. Interaction of Many Discrete States with Many Continua. Phys. Rev. B 1976, 15, 2961-2969.

(47) Wimmer, E.; Krakauer, H.; Weinert, M.; Freeman, A. J. Phys. Rev. B 1981, 24, 864.

(48) Jansen, H. J. F.; Freeman, A. J. Total-Energy Full-Potential Linearized Augmented-Plane-Wave Method for Bulk Solids: Electronic and Structural Properties of Tungsten. Phys. Rev. B 1984, 30, 561569.

(49) Dressel, M.; Grüner, G. Electrodynamics of Solids; Cambridge University Press: Cambridge, UK, 2002.

(50) Matsuishi, S.; Toda, Y.; Miyakawa, M.; Hayashi, K.; Kamiya, T.; Hirano, M.; Tanaka, I.; Hosono, H. High-Density Electron Anions in a Nanoporous Single Crystal: $\left[\mathrm{Ca}_{24} \mathrm{Al}_{28} \mathrm{O}_{64}\right]^{4+}\left(4 \mathrm{e}^{-}\right)$. Science 2003, 301, 626-629.

(51) Liu, H. L.; Quijada, M.; Romero, D. B.; Tanner, D. B.; Zibold, A.; Carr, G. L.; Berger, H.; Forró, L.; Mihaly, L.; Cao, G.; et al. Drude Behavior in the Far-Infrared Conductivity of Cuprate Superconductors. Ann. Phys. (Leipzig) 2006, 15, 606-618. 